\title{
Diversity and evolution of Hunter-Schreger Band configuration in tooth enamel of perissodactyl mammals
}

Wighart Von Koenigswald, Luke T. Holbrook, and Kenneth D. Rose

Acta Palaeontologica Polonica 56 (1), 2011: 11-32 doi: http://dx.doi.org/10.4202/app.2010.0021

Four different Hunter-Schreger Band (HSB) configurations were observed in the teeth of fossil and extant Perissodactyla. This variability exceeds that observed in Artiodactyla or Proboscidea. The four HSB configurations represent two different evolutionary pathways. Transverse HSB found in many mammalian taxa outside the Perissodactyla represents the most primitive HSB configuration. It occurs in several primitive perissodactyl families and is retained in Palaeotheriidae and extant Equidae. Curved HSB evolved from transverse HSB and occurs in Tapiridae, Helaletidae, and Lophiodontidae, as well as in Ancylopoda and Titanotheriomorpha. This likely indicates independent evolution of curved HSB in two or more lineages, but the number of instances of parallelism of this configuration is obscured by uncertainty in the relationships among these taxa and by a lack of data for some important basal taxa. A second evolutionary pathway leads from transverse HSB via compound HSB to vertical HSB. Compound HSB were detected in Hyrachyidae, Deperetellidae, and the early rhinocerotid Uintaceras. Vertical HSB configuration characterizes the molar dentition of other Rhinocerotidae, Hyracodontidae, Indricotheriidae, andAmynodontidae. Often, the incisors of rhinocerotids retain traces of compound HSB. Thus theHSB configuration reflects phylogenetic relationships to some degree. The selective value of themodified HSB configurations is interpreted functionally as amechanismto reduce abrasion duringmastication, assuming that the perpendicular intersection of prismswith the actual grinding surfaces resists wear better than prisms running parallel to the occlusal surface.

Key words: Mammalia, Perissodactyla, Hunter-Schreger Bands, HSB, tooth enamel microstructure, functional adaptation, phylogeny.

Wighart von Koenigswald [Koenigswald@uni-bonn.de], Steinmann Institut (Paleontology), University of Bonn, Nussallee 8 D-53115 Bonn, Germany; Luke

T. Holbrook [holbrook@rowan.edu], Department of Biological Sciences, Rowan University, Glassboro, NJ 08028 USA; Kenneth D. Rose [drose@ jhmi.edu], Center for Functional Anatomy and Evolution, Johns Hopkins University School of Medicine, Baltimore, MD 21205 USA. 
This is an open-access article distributed under the terms of the Creative Commons Attribution License (for details please see creativecommons.org), which permits unrestricted use, distribution, and reproduction in any medium, provided the original author and source are credited.

For Full text $(1,151.4 \mathrm{kB})$ 